\title{
$K$-scrambling in a near-symmetric top molecule containing an excited noncoaxial internal rotor
}

\author{
Juan Ortigoso ${ }^{\mathrm{a})}$ \\ Instituto de Estructura de la Materia, Consejo Superior de Investigaciones Científicas, Serrano 121, \\ 28006 Madrid, Spain \\ Jon T. Hougen ${ }^{b)}$ \\ Optical Technology Division, National Institute of Standards and Technology, Gaithersburg, \\ Maryland 20899-8441
}

(Received 18 January 2000; accepted 24 March 2000)

\begin{abstract}
Classical trajectories on rotational energy surfaces and coherent-state quantum projections have been used to study an asymmetric-top molecule containing a freely rotating internal symmetric top whose symmetry axis is not coincident with a principal axis of the molecule. Stationary points on the rotational energy surface, which strongly influence the trajectories, increase in number from two to four to six as $J / n$ increases from zero to infinity (where $J$ is the total and $n$ is the free-internal-rotor angular momentum). For some $J / n$ values trajectories can arise that sample a large fraction of $K$ values (where $K$ is the $z$-axis projection of $J$ ), corresponding in quantum wave functions to extensive $K$ mixing in the symmetric-top basis set $|J, K\rangle$. When such mixing cannot be made small for any choice of $z$ axis, we call it $K$ scrambling. For typical values of the torsionrotation coupling parameter $\rho$, rotational eigenfunctions for a given $J$ and torsional state turn out to be quite different from eigenfunctions for the same $J$ in some other torsional state. Nonzero rotational overlap integrals are then distributed among many rotational functions for each $\left(n, n^{\prime}\right)$ pair, which may, in turn, contribute to internal rotation enhancement of intramolecular vibrational energy redistribution. We have also examined near-free-rotor levels of our test molecule acetaldehyde, which arise for excitation of ten or more quanta of methyl group torsion, and find that barrier effects do not change the qualitative picture obtained from the free-rotor treatment. (c) 2000 American Institute of Physics. [S0021-9606(00)00823-0]
\end{abstract}

\section{INTRODUCTION}

The spectroscopy and dynamics of molecules containing an internal rotor is an old topic, which is nevertheless still far from being completely understood. Although the basic theory for understanding their microwave spectra was reviewed 40 years ago by Lin and Swalen, ${ }^{1}$ numerous contributions have been published in the last few years that deal with different topics, such as axis systems, ${ }^{2-4}$ Hamiltonians, ${ }^{5,6}$ analysis of high-resolution molecular spectra, ${ }^{7}$ or studies of the classical mechanics of these systems ${ }^{8,9}$ and its quantum manifestations. ${ }^{10}$

We have been interested in the problem of finding meaningful labels for torsion-rotation quantum states of the prototype molecule acetaldehyde $\left(\mathrm{CH}_{3} \mathrm{CHO}\right)$ for the purpose of assigning unambiguously high-resolution molecular spectra, and this paper deals with some aspects of that problem above the barrier. Labeling problems arise for torsion-rotation wave functions because the internal rotation of the methyl group is strongly coupled to the overall rotation (primarily via torsion-rotation Coriolis coupling for rotors with $C_{3 v}$ symmetry). This causes extensive basis set mixing and ambiguous eigenvectors (in the sense of an inability to find one clearly dominant basis function in each eigenvector).

\footnotetext{
a)Electronic mail: ortigoso@alpharaf.iem.csic.es

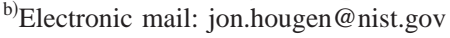

In our previous studies, confined almost exclusively to energy levels below the barrier, we employed tools based on classical and semiclassical concepts such as Poincaré surfaces of section, ${ }^{10}$ rotational energy surfaces (RES), ${ }^{11,12}$ and phase-space representations of quantum states obtained by projecting wave functions over angular-momentum coherent states. ${ }^{10,13-15}$ In particular, we discussed the RES concept for asymmetric-top molecules containing an internal rotor $^{12}$ and proposed a simple expression to represent such surfaces below the barrier [see Eq. (5) of Ref. 12 and Eq. (3) of Ref. 15]. The mixing of rotation and torsion as the total angular momentum $J$ increases distorts the surfaces by creating new stationary points (maxima, minima, and saddles) and new separatrices, which, in turn, give rise to complicated rotational trajectories in the low- $K_{a}$ region ${ }^{15}$ ( $K_{a}$ is the projection of $J$ along the $z=a$ axis). We found below the top of the barrier to internal rotation (i.e., for torsional states $v_{t}=0,1$, and 2 of acetaldehyde), that eigenvectors exhibiting a complicated rotational basis-set composition when viewed in the rho-axis (RAM) Hamiltonian basis $\operatorname{set}^{2}$ can be greatly simplified ${ }^{12,15}$ by rotating to a new axis system defined by an appropriate stationary point of the RES. We also showed using coherent-state projections ${ }^{15}$ that classical trajectories on appropriate rotational energy surfaces give faithful representations of these quantum wave functions. Irregularities in the rotational structure of torsional states below the barrier were explained in terms of extra separatrices in the rotational 
energy surface. The associated eigenvectors have quantumphase-space representations with unusually high densities at saddle points of the RES.

Recently, an in-depth study of the classical dynamics of toluene (characterized by a sixfold barrier and a symmetrictop rotor coaxial with one principal axis) was published by Vivian, Lehn, and Frederick. ${ }^{9}$ They found a limited degree of weak chaos for trajectories near the top of the barrier, in agreement with an earlier similar finding in acetaldehyde, ${ }^{10}$ and delineated several clear differences in behavior between chaos in an internal rotor system and chaos in coupled anharmonic oscillator systems. They also observed a shifting of the stable and unstable axes of rotation for the case of free methyl torsion, and discussed changes in the rotational dynamics with increasing excitation of the internal rotor.

In the present paper we seek to understand the rotational dynamics of acetaldehyde near the free-rotor limit, which is closely related to Sec. IV A of Ref. 9 (though acetaldehyde has a threefold barrier and a noncoaxial symmetric-top rotor). We have characterized wave functions of acetaldehyde and find that for high enough excitation of the internal rotation of the methyl group (e.g., 11 or more quanta of torsion in acetaldehyde, i.e., $E=900 \mathrm{~cm}^{-1}$ or higher), some rotation-torsion states correspond closely to free internal rotation states, while (somewhat unexpectedly) the overall rotation motion classically samples a considerable part of the available phase space. Quantum mechanically this behavior gives rise to $K$-scrambled states, i.e., to wave functions strongly mixed in a symmetric-top basis set, regardless of the axis system used.

We believe that $K$-scrambled states in excited internal rotor molecules can participate in the enhancement of essentially irreversible intramolecular vibrational redistribution (IVR) via a mechanism similar to the vibrationally induced rotational-axis-switching mechanism (VIRAS) proposed by Li, Ezra, and Philips ${ }^{16}$ to explain vibrational mode coupling and enhancement of IVR in molecules containing internal rotor groups. Studies on the role of $K$ scrambling in the enhancement of energy transfer in molecules without internal rotation were carried out by Nathanson and McClelland, who proposed ${ }^{17}$ using the reduction of fluorescence polarization to characterize the transition from regular to statistical rotation and to explore the breakdown ${ }^{18}$ of the rigid body description of rotational motion as a result of strong Coriolis interactions.

\section{THE FREE-ROTOR LIMIT FOR AN ASYMMETRIC TOP MOLECULE WITH A NONCOAXIAL SYMMETRIC-TOP INTERNAL ROTOR}

\section{A. Free-rotor rotational energy surfaces}

Rotational energy surfaces (RESs) ${ }^{11}$ are radial plots of the energy of a molecule as a function of the direction of the total angular momentum in the molecule-fixed frame. They are based on the adiabatic separation of molecular rotation from all other degrees of freedom, a criterion that is well satisfied for small-amplitude torsional oscillations deep in the potential well. Near the top of the barrier to internal rotation this adiabatic separation is not a good approxima- tion, since the quantum eigenfunctions often contain significant contributions from many torsional and rotational states, and the optimum definition of a rotational energy surface is thus not clear. In the free-rotor limit, however, adiabatic separation between torsion and rotation is recovered again, in the sense that wave functions are well represented by a single product of a torsional part and a rotational part.

The classical dynamics of a rotating system can be represented by trajectories on RESs. ${ }^{11}$ Since these trajectories are strongly influenced by the number, type, and location of stationary points on the RES, we first investigate stationary points on RESs for asymmetric rotors with a noncoaxial symmetric-top free internal rotor. Quantum eigenvectors of acetaldehydelike molecules can be classified as $A$ or $E$ according to their symmetry in the $G_{6}$ symmetry group, and the appropriate rotational energy surfaces are different for these two types of wave functions. As the free-rotor limit is approached, the signed value of $n$ becomes a good quantum number for $E$-symmetry states $\left(|n\rangle=e^{+i n \alpha}\right.$, where $\alpha$ is the internal rotation angle), but for $A$ states $|+n\rangle$ and $|-n\rangle$ basis functions must be equally mixed to satisfy the $A_{1}$ and $A_{2}$ symmetry requirements of $G_{6}$. To avoid this complication we consider only $E$-symmetry states here.

Free-rotor $E$-symmetry RESs have the following simple form (in the RAM axis system ${ }^{7}$ ) as a function of the two spherical polar angles $\theta$ and $\chi$ :

$$
\begin{aligned}
E(n ; \theta, \chi)= & F\left(n-\rho J_{q} \cos \theta\right)^{2}+\left(V_{3}+V_{6}\right) / 2 \\
& +A J_{q}^{2} \cos ^{2} \theta+B J_{q}^{2} \sin ^{2} \theta \cos ^{2} \chi+C J_{q}^{2} \\
& \times \sin ^{2} \theta \sin ^{2} \chi-2 D_{a b} J_{q}^{2} \sin \theta \cos \theta \cos \chi,
\end{aligned}
$$

where $F, \rho, A, B, C, D_{a b}, V_{3}$, and $V_{6}$ are torsional and rotational spectroscopic constants given in Table I of Ref. 12. Equation (1) is obtained by substituting the classical limit for the angular momentum components in the quantum mechanical Hamiltonian of Eq. (2) below. The two barrier constants $V_{3}$ and $V_{6}$ are retained as constant energy offsets in this free rotor Hamiltonian because we approach the free rotor limit in the numerical calculations of Sec. III by going to energy levels well above the barrier, rather than by decreasing the barrier height to zero. For comparison with quantum results, $J_{q}$ in Eq. (1) must be given values of the form $J_{q}=[J(J+1)]^{1 / 2}$.

The stationary points for the RAM Hamiltonian in Eq. (1) depend only on the ratio $J_{q} / n$. For low values of $J_{q} / n$ (corresponding to the limiting case of a gyroscope little affected by the slow overall rotation of its supporting host structure) there are only two stationary points; for intermediate values of $J_{q} / n$ there are four; and for high values (corresponding to the limiting case of a rigid rotor little affected by the slow rotation of its internal gyroscope) there are six stationary points. This behavior is illustrated schematically by the three circles in Fig. 1. The first circle shows the position of the maximum $(\bigcirc$ or $M)$ and minimum $(\bigcirc$ or $m)$ in the $x z$ plane of a RES for acetaldehyde with $J_{q} / n=0.55$ and 


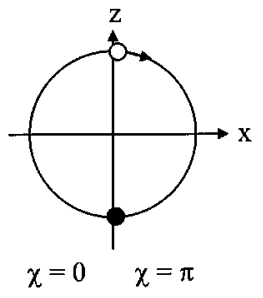

(a)

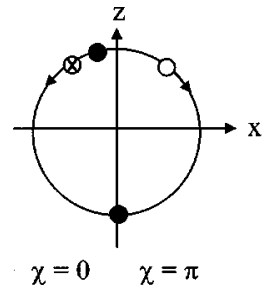

(b)

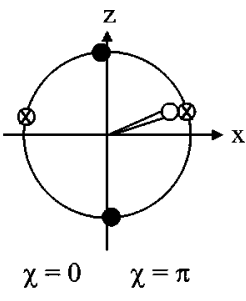

(c)
FIG. 1. Behavior of the stationary points on the RES of Eq. (1) for three values of $J_{q} / n$, illustrated schematically for the example molecule acetaldehyde on a circle in the $x z$ plane using the conversion from Cartesian to spherical polar coordinates given in Eq. (1) of Ref. 12, so that $\chi=0$ and the $-x$ axis lie to the left, and $\chi=\pi$ and the $=+x$ axis lie to the right of the vertical $z$ axis in this figure. (a) For $J_{q} / n=0.55$ the RES has only two stationary points: a maximum $(\bullet)$ and a minimum $(\bigcirc)$. These points both lie in the $x z$ plane, near the south and north pole, respectively. The minimum moves rapidly in the direction of the arrow as $J_{q} / n$ increases. (b) For $J_{q} / n=1.35$ the RES has four stationary points, again all lying in the $x z$ plane. The earlier minimum has migrated toward the equator. A new maximum $(\bullet)$ and saddle $(\otimes)$ pair were created near the north pole (with $\chi$ $=0)$ when $J_{q} / n$ increased through the bifurcation value $\left(J_{q} / n\right)_{c}=1.29$. (c) For $J_{q} / n=4.35$ the RES has six stationary points, two maxima and two saddles lying in the $x z$ plane and two minima symmetrically placed above and below it. The new minimum and saddle pair were created in the $x z$ plane at the position of the existing $\chi=\pi$ minimum when $J_{q} / n$ reached $\left(J_{q} / n\right)_{c}=3.48$. The new saddle remained in the $x z$ plane as $J_{q} / n$ increased, but the two minima began migrating toward their final $\left(J_{q} / n \rightarrow \infty\right)$ locations on the $+y$ and $-y$ axes.

$(1 M, 0 s, 1 m)$. The second circle, with $J_{q} / n=1.35$ and $(2 M, 1 \mathrm{~s}, 1 \mathrm{~m})$, shows the migrated positions in the $x z$ plane of the original maximum and minimum, as well as positions of the new maximum and saddle $(\otimes$ or $s)$ created at the first bifurcation when $J_{q} / n=\left(J_{q} / n\right)_{c}=1.29$. The third circle, with $J_{q} / n=4.35$ and $(2 M, 2 s, 2 m)$, shows the migrated positions in the $x z$ plane of the previous saddle and two maxima, as well as the location of a saddle created at the second bifurcation when $J_{q} / n=\left(J_{q} / n\right)_{c}=3.48$. The original minimum, and its partner created at $\left(J_{q} / n\right)_{c}=3.48$, are located symmetrically above and below the $x z$ plane of the third circle, as required by the fact that the RES of Eq. (1) for $n \neq 0$ has an $x z$ plane of symmetry. The saddles, however, are not symmetrically related and give rise to two distinct separatrices here, in contrast to the single separatrix required by symmetry in the coaxial case treated in Ref. 9. In any case, as $J_{q} / n$ approaches infinity the various stationary points continue to migrate, until the familiar rigid rotor pattern of maxima on the principal $a$ axis (near the RAM $z$ axis), saddles on the principal $b$ axis (near the RAM $x$ axis), and minima on the principal $c$ axis (RAM $y$ axis) is reached.

We now examine the classical RES trajectories in more detail. Figure 2 shows four projections onto a plane of trajectories on $\mathrm{RAM}^{2} \mathrm{RESs}^{10}$ corresponding to different $J_{q} / n$ $>0$ values in the free internal rotor RESs defined by $\left[E(n ; \theta, \chi)-\frac{1}{2}\left(V_{3}+V_{6}\right)\right] / n^{2}$ from Eq. (1). The first panel corresponds to a RES with $(1 M, 0 s, 1 \mathrm{~m})$, similar to that depicted in the first circle of Fig. 1, and all trajectories in this $J_{q} / n=0.5$ panel exhibit a fairly flat projection of the angular momentum vector onto the RAM $a$ axis [whose negative end passes close to the maximum near the south pole, as in Fig. 1(a)]. These flat trajectories imply quantum mechanically
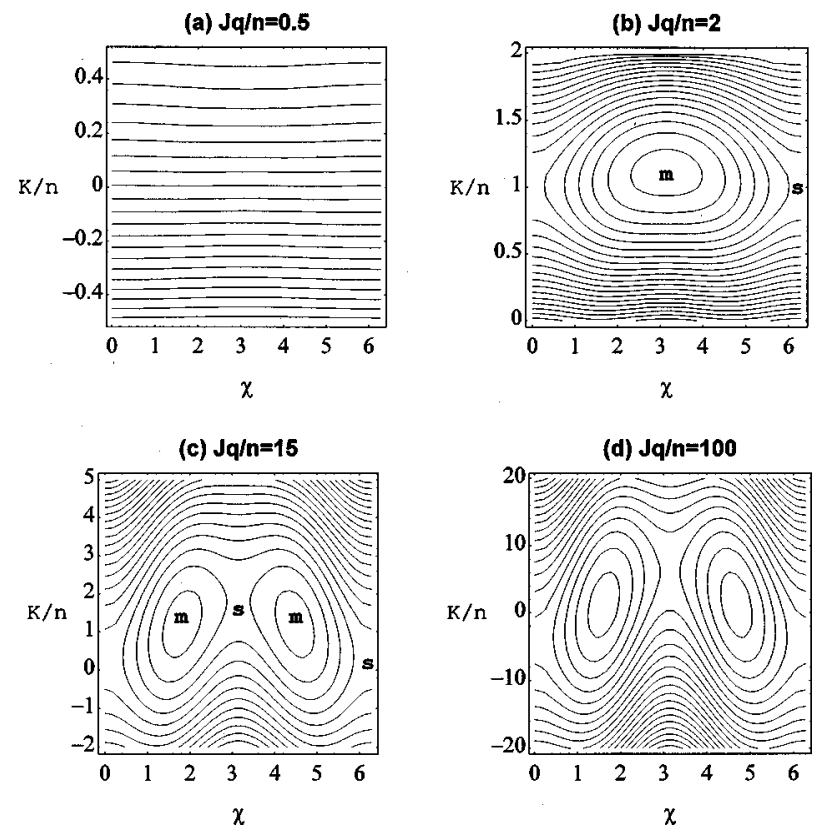

FIG. 2. Classical trajectories on the RES of Eq. (1) for four values of $J_{q} / n$, displayed by projecting the RES from its center onto a square in the plane of the paper. (Note that distance relationships on the RES can be grossly distorted by this projection, e.g., the two points representing the north and south pole of the RES become two straight lines across the top and bottom of each panel, circles on the equator often appear as rather eccentric ellipses, etc.) Panel (a) corresponds to a RES with low $J_{q} / n$ and $(1 M, 0 s, 1 m)$, as in Fig. 1(a). Trajectories in this panel are nearly flat, indicating that $K_{a}^{\mathrm{RAM}}$ is a rather good quantum number. ${ }^{12}$ Panel (b) corresponds to a RES with intermediate $J_{q} / n$ and $(2 M, 1 s, 1 m)$, as in Fig. 1(b). Trajectories enclosing the minimum $(m)$ correspond to quantum states with extensive $K$ scrambling. Panel (c) corresponds to high $J_{q} / n$ and $(2 M, 2 s, 2 m)$, as in Fig. 1(c). Since the two saddle points lie at different energies, trajectories arise that simultaneously enclose the two equivalent minima $(m)$ and the lower-energy saddle $(s)$. Such trajectories also correspond to quantum states with extensive $K$ scrambling. Panel (d) corresponds to very high $J_{q} / n$, i.e., nearly to the rigid asymmetric rotor limit at $J_{q} / n=\infty$. It is shown for comparison in the same rho-axis system as the other three panels, even though it would have a more symmetrical appearance if it were transformed to the principal axis system, where the maxima, saddles, and minima lie nearly on the $\pm z$, $\pm x$, and $\pm y$ axes, respectively. Each trajectory in the rigid rotor limit encloses only one maximum or minimum; no trajectory encloses a saddle.

that $K_{a}^{\mathrm{RAM}}$ is a good quantum number for the corresponding low- $\left(J_{q} / n\right)$ wave functions.

The second panel in Fig. 2 corresponds to a RES with $(2 M, 1 s, 1 m)$, as depicted in the second circle of Fig. 1. It displays a new class of trajectories in the region of $K / n$ $\approx 1$, which avoid the saddle $(s)$ at $\chi=0$ and enclose the minimum $(m)$ at $\chi=\pi$. (For RESs with negative $n$ values, such trajectories would arise in the negative $K / n$ region of the southern hemisphere.) At first glance, these $J_{q} / n=2$ trajectories are reminiscent of those near the equator on the RES of a rigid asymmetric top, but two qualitative differences are present. First, there is only one saddle at $\chi=0$, not two saddles symmetrically located at $\chi=0$ and $\pi$. Second, the saddle and minimum are not located near the equator, but rather at a latitude farther north, where the distance around the RES is expected to be smaller than at the equator. This short-distance effect is even more pronounced in the present problem, as can be seen from the pearlike shape of the $J_{q} / n=1.96$ RES shown in Fig. 3. (Distances on the RES are 


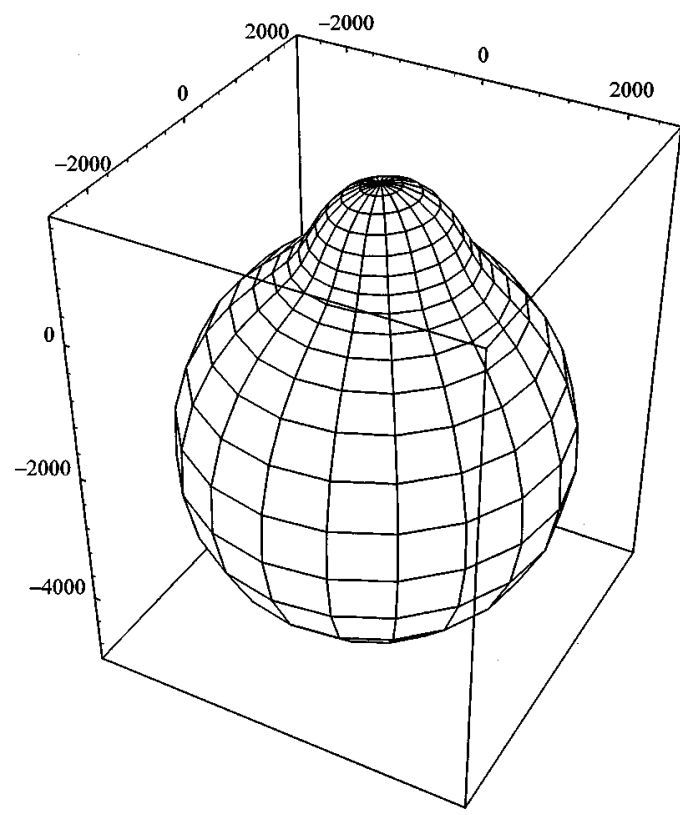

FIG. 3. A free-rotor RES for acetaldehyde obtained from the Hamiltonian in Eq. (1) with $n=13$ and $J_{q}=[25(25+1)]^{1 / 2}$. This RES has $(2 M, 1 s, 1 m)$, and corresponds closely to the projection in Fig. 2(b). Note the pronounced difference between the northern and southern hemispheres, arising because of the torsion- $K$-rotation Coriolis coupling and the lack of an $x y$ plane of symmetry. The existence of an $x z$ plane and the lack of a $y z$ plane of symmetry cannot easily be seen in this diagram.

important because quantization requirements for classical trajectories define minimum areas that must be encircled on the RES.)

Since some of the trajectories in the second panel of Fig. 2 enclose the minimum of the RES, it is interesting to rotate the RES using Eq. (3) of Ref. 15, such that $J_{\chi}$ becomes the projection of the angular momentum along an axis drawn from the origin to the minimum. This method was used earlier to obtain well defined ${ }^{12}$ or better defined ${ }^{15} K$ labels for eigenvectors that showed some degree of mixing in the original $|J, K\rangle_{\text {RAM }}$ basis set. Surprisingly, a trajectory enclosing the minimum in Fig. 2(b), when viewed on a rotated RES as illustrated in Fig. 4(c), samples an even bigger $J_{\chi}$ region than in the original axis system, indicating that the $K$ mixing here has a true physical origin and is not simply caused by a bad choice of axis system in which the Hamiltonian is written. We use the term $K$ scrambling to denote extensive $K$ mixing that cannot be eliminated by any rotation of the Cartesian axes.

Figure 2(c) illustrates trajectories in the regime of six stationary points for a $J_{q} / n$ value well after creation of the third pair, so that the two minima have migrated to positions near $\chi= \pm \pi / 2$. As mentioned, the two saddles are not symmetrically related, and there are thus two separatrices (at two different energies) in this $J_{q} / n=15$ figure. As a result, in addition to trajectories enclosing one or the other of the two minima, it is also possible to have trajectories that enclose both minima and the lower-energy saddle and separatrix. Based on our previous experience, ${ }^{15}$ we expect such states to show extensive $K$ scrambling.

Figure 2(d) illustrates trajectories for the $J_{q} / n=100$ case of six stationary points that have all migrated to near their rigid-rotor positions on the positive and negative side of each principal axis. (These trajectories would look more symmetrical if they were viewed in the principal axis system rather than in the RAM system, as illustrated in Fig. 1 of Ref. 15.) Figure 2(d) (at the resolution used here) closely resembles the $J_{q} / n \rightarrow \infty$ rigid-rotor limit, where the two saddles have the same energy. Then only a single separatrix is present, which divides the RES into four noncommunicating regions, so that each trajectory encloses exactly one maximum or one minimum, and no trajectory encloses a saddle (the Poincaré index of a closed curve ${ }^{19}$ must be 1).

It is interesting to compare the results above with analogous results for an asymmetric-top molecule with an internal rotor whose symmetric-top axis is parallel to the principal inertial $a$ axis of the molecule, as given in Table II of Ref. 9. [This coaxial case can be obtained by setting $D_{a b}=0$ and $F=A / \rho(1-\rho)$ in Eq. (1) and using the numerical value $A /(1-\rho)$ in place of $A$ in Table II of Ref. 9.] As $J_{q}$ increases from near zero to infinity in the coaxial case, the number of stationary points again changes from two to four to six, but for a very near prolate rotor like acetaldehyde, quantitative differences arise because the intermediate range of $J_{q} / n$ values corresponding to four stationary points is much smaller for the coaxial case $^{9}\left(1.12 \leqslant J_{q} / n \leqslant 1.14\right.$ for acetaldehyde with $\left.D_{a b}=0\right)$ than for the noncoaxial case $\left(1.29 \leqslant J_{q} / n\right.$ $\leqslant 3.48$ for acetaldehyde with $D_{a b}=-0.123 \mathrm{~cm}^{-1}$ ). Qualitative differences also arise because the RES for the coaxial rotor problem of Ref. 9 has both an $x z$ and a $y z$ plane of symmetry, whereas that for the noncoaxial rotor considered here has only an $x z$ plane of symmetry. All stationary points are thus symmetrically paired in the coaxial case, except those at the north and south poles, whereas all stationary points are symmetrically paired in the noncoaxial case, except those on the intersection of the RES with the $x z$ plane. This in turn leads to the fact that bifurcations in the coaxial case are restricted to the poles, whereas bifurcations in the noncoaxial case are restricted only to the $x z$ plane. Finally, the higher symmetry of the coaxial RES prevents pairs of saddle points with unequal energies from arising, which in turn prevents a second separatrix and trajectories that enclose a separatrix [as in Fig. 2(c)] from arising.

\section{B. Free-rotor quantum wave functions}

Free-rotor states arise in molecules with an internal rotor at high energies where effects of the barrier can be neglected. Free-rotor states could thus be studied by setting up a Hamiltonian in which all barrier terms were artificially removed. In this paper, however, for easy comparison between free-rotor wave functions and actual acetaldehyde wave functions, we set up the matrix of the quantum Hamiltonian,

$$
\begin{aligned}
H= & F\left(p_{\alpha}-\rho J_{z}\right)^{2}+\frac{1}{2}\left[V_{3}(1-\cos 3 \alpha)+V_{6}(1-\cos 6 \alpha)\right] \\
& +A J_{z}^{2}+B J_{x}^{2}+C J_{y}^{2}+D_{a b}\left(J_{x} J_{z}+J_{z} J_{x}\right)
\end{aligned}
$$

in the product basis set of free internal rotor functions $e^{i n \alpha}$ and symmetric-top rotational functions $|J, K\rangle$, and set to zero all matrix elements of the barrier terms off-diagonal in $n$, while retaining their diagonal (constant) energy contribu- 
(a)

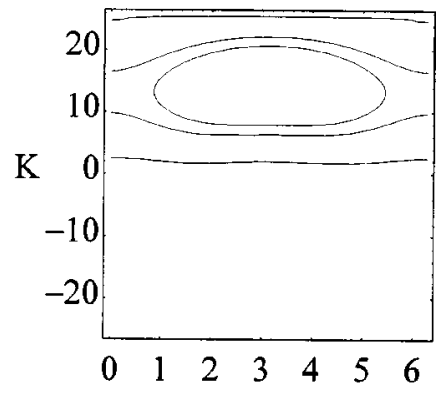

$\chi$

\section{(b)}

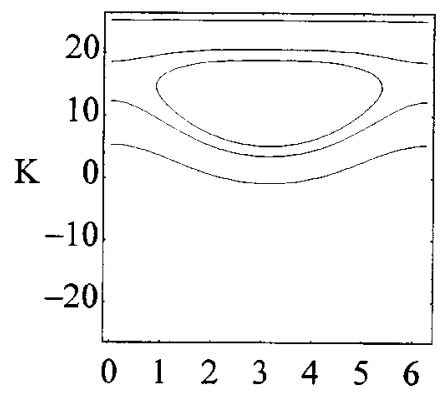

$\chi$

(c)

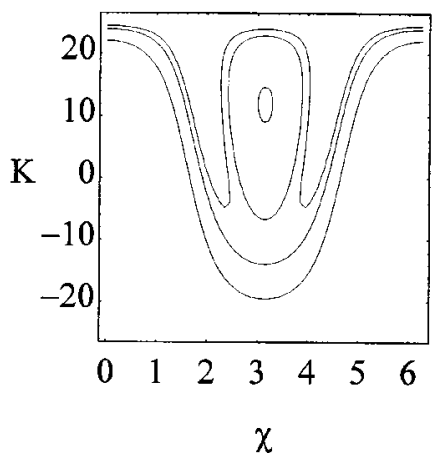

FIG. 4. Five trajectories, at three different energies, on the RES for $n=13$ and $J_{q}=[25(25+1)]^{1 / 2}$, projected onto a plane for three different choices of $z$ axis (i.e., of the north pole for the RES). (a) A projection using the RAM $z$ axis. The flat outer trajectories near $K=+2$ and +24 correspond to an energy of $E=1582.66 \mathrm{~cm}^{-1}$ and sharply defined $K_{a}^{\text {RAM }}$ quantum numbers. They encircle the maxima near the north and south pole, respectively. The two middle trajectories correspond to $E=1360.38 \mathrm{~cm}^{-1}$, and also encircle one of the two maxima. The inner ellipsoidal trajectory corresponds to $E$ $=1300.16 \mathrm{~cm}^{-1}$ and encloses the minimum located near its center. (b) A projection of these same trajectories after the RES $z$ axis has been rotated slightly to pass exactly through the nearby maximum. The two upper trajectories have become flatter (indicating better defined $K_{a}$ quantum numbers), but the lower three trajectories have become less flat. (c) A projection of these same trajectories with the RES $z$ axis passing though the minimum. The rather flat trajectories near $K=+24$ and +18 in Fig. 2(b) give rise to the smaller and larger ellipse in Fig. 2(c) respectively, which actually represent nearly circular trajectories (around a maximum) whose shapes have been distorted because of the angular scale difference in the two dimensions of the projection square. The ellipse in Fig. 2(b) gives rise in 2(c) to the clamplike trajectory partially enclosing the larger ellipse. This trajectory illustrates the main point of this figure: The sampling of $K$ values for this trajectory around the minimum is significantly smaller in Fig. 2(a) than in Fig. 2(c), even though a naïve application of classical trajectory ideas would suggest that the opposite is to be expected. The bottom two trajectories in Fig. 2(b) give rise in 2(c) to the inverted bell curves, which are of no further interest here. tions. [Note the change of sign in the last term of Eq. (2) with respect to the classical expression in Eq. (1) because of Eqs. (1) in Ref. 12.] This procedure leads to solving an effective rigid-rotor Hamiltonian for each $n$ value, and produces torsion-rotation wave functions of the form

$$
\psi_{\mathrm{tr}}=e^{i n \alpha} \sum_{K} c_{n K}|J, K\rangle,
$$

where $n$ is a rigorously good quantum number. These wave functions can be directly related to the RES trajectories shown in the previous section as follows.

Table I gives $K$ compositions for quantum wave functions corresponding to three of the five trajectories shown in Fig. 4. The first three columns correspond to the ellipse in Fig. 4(a) and give coefficients in the quantum wave function greater than 0.1 for $K$ (in parentheses) basis functions (i) in the RAM axis system of Fig. 4(a), (ii) in the $z \rightarrow M$ axis system of Fig. 4(b), i.e., in the system obtained by rotating $z$ to the direction defined by the maximum near the north pole in the RAM system [see also Fig. 1(b)], and (iii) in the $z$ $\rightarrow m$ system, where $m$ is the minimum enclosed by the ellipse in Fig. 4(a). Note, as expected from the classical trajectories in Fig. 4(c), that this quantum wave function has more $K$ components larger than 0.1 in the $z \rightarrow m$ system than in the original RAM system. The second three columns give analogous coefficients for the wave function corresponding to the second trajectory from the top in Figs. 4(a) and 4(b). Its $K$ composition sharpens up considerably in the $z \rightarrow M$ system. The last three columns give analogous coefficients for the wave function associated with the bottom trajectory in Figs. 4(a) and 4(b). As expected from Fig. 4, this wave function has its sharpest $K$ composition in the original RAM axis system. Unexpectedly, however, this wave function (which lies below the separatrix) becomes somewhat less sharp when expressed in an axis system with $-z$ passing through the maximum near the south pole (not shown in Table I).

The wave functions of Table I are visualized in Fig. 5 by projecting them over angular momentum coherent states (see Ref. 15 for a recipe). Figure 5(a) shows the wave function corresponding to the elliptical trajectory around the minimum in Fig. 4(a). The concentration of density at the extremes of the trajectory, instead of being distributed evenly all around the ellipse, (cf. Figs. 1, 4, and 5 of Ref. 15) was at first surprising. Such a density pattern is reminiscent of the patterns seen at turning points of vibrational motion, and probably implies that the system does not spend much of its time on the nearly flat top and bottom portions of the ellipse in Fig. 4(a), but instead spends most of its time making the turns at the ends of the long axis of the ellipse. This interpretation helps explain the extensive $K$ scrambling shown in Table I. Indeed, if the reverse were true, one might expect a strongly bimodal distribution of coefficients, i.e., one peaked much more sharply in the narrow range of $K$ values along each of the two long, nearly flat parts of the elliptical trajectory. Figures 5(b) and 5(c) correspond to the patterns expected for the arched second and rather flat last trajectories in Fig. 4(a), and thus have no special interest, except to show once again that trajectories on the RES give faithful representations of rotational wave functions. 


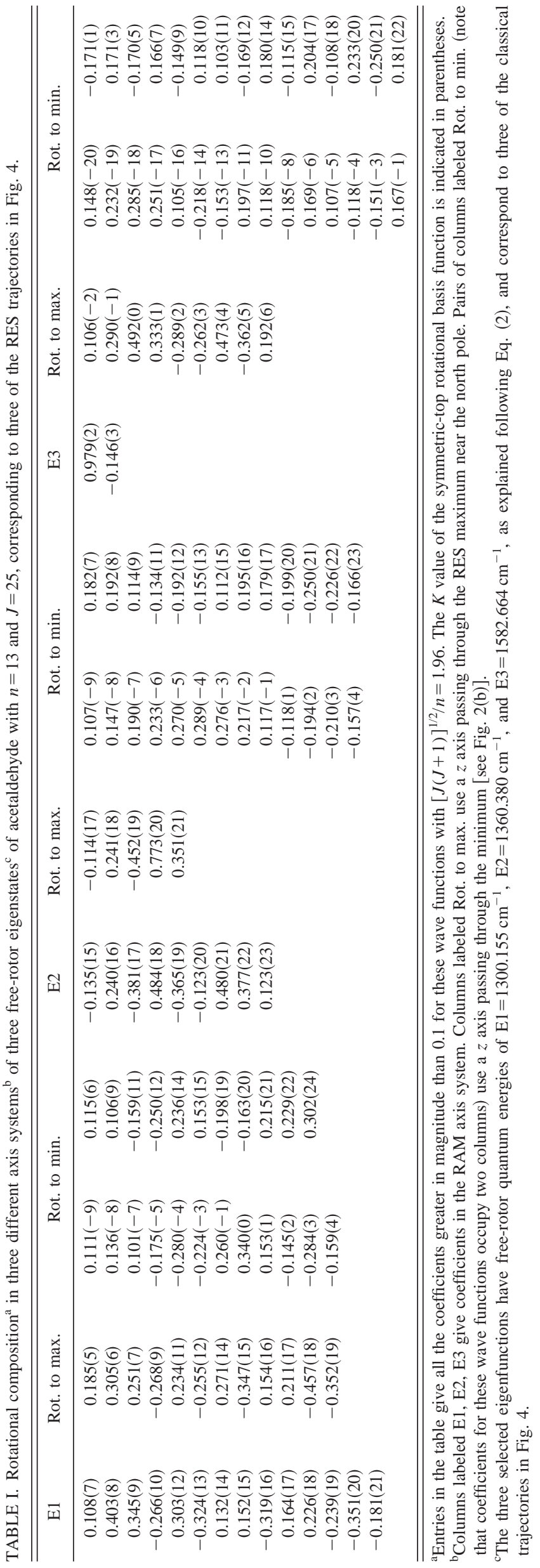

(a)

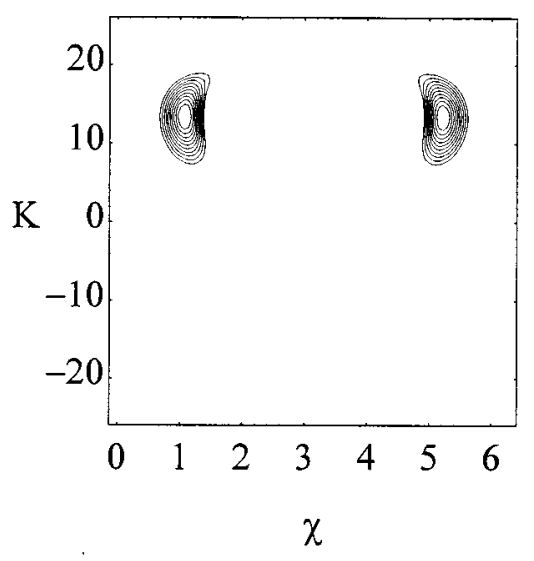

(b)

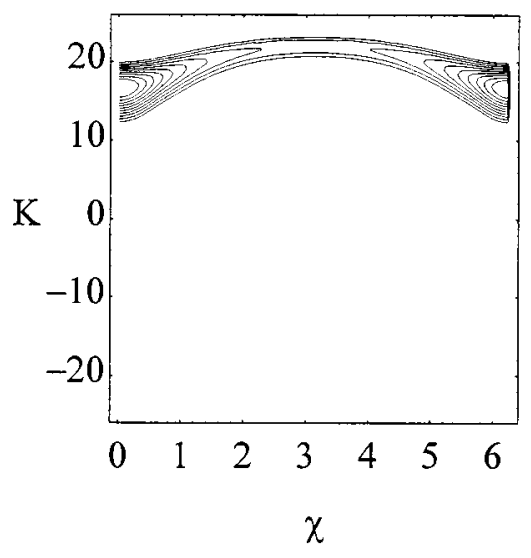

(c)

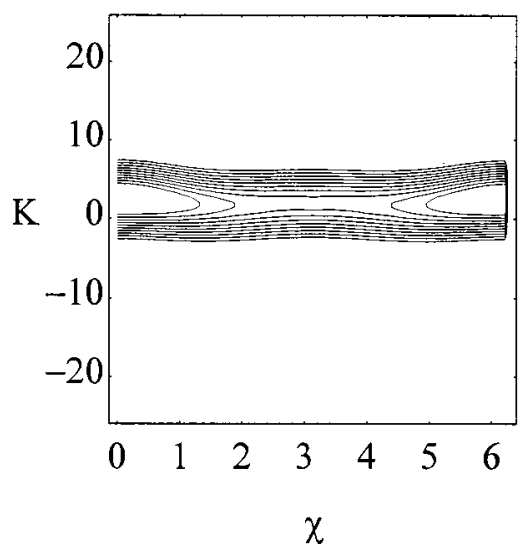

FIG. 5. Coherent state projections of quantum wave functions corresponding to (a) the ellipse in Figs. 4(a) and 4(b), and E1 in Table I; (b) the second (nearly flat) trajectory from the top in Figs. 4(a) and 4(b), and E2 in Table I; and (c) the bottom trajectory in Figs. 4(a) and 4(b), and E3 in Table I. Density contours in each panel are plotted down to about one-third of the maximum density. (a) illustrates the unusual density pattern concentrated at the ends of the ellipse, rather than at the minimum near its center, a phenomenon probably connected to the extensive $K$ scrambling in this state. (b) and (c) show no unusual features, but they do confirm that classical RES trajectories give faithful representations of quantum wave functions.

Since the location of the RES minimum giving rise to $K$-scrambled states migrates rapidly with quantum number $n$ for a particular $J$ value, the $K$ composition of associated wave functions also changes rapidly with $n$ for given $J$. This 
TABLE II. Number of free-rotor torsion-rotation states (as a function of the number of torsional quanta $n$ ) for which the rotational part of the corresponding wave function has an overlap larger than 0.1 with the rotational part of the three $n=13$ free rotor torsion-rotation eigenstates shown in Table I.

\begin{tabular}{rrrr}
\hline \hline$n$ & E1 & E2 & E3 \\
\hline 4 & 14 & 6 & 8 \\
7 & 16 & 6 & 4 \\
10 & 10 & 4 & 1 \\
13 & 1 & 1 & 1 \\
16 & 13 & 8 & 1 \\
19 & 15 & 10 & 1 \\
22 & 14 & 11 & 1 \\
\hline \hline
\end{tabular}

implies that the rotational part of $K$-scrambled wave functions for given $J$ and $n$ will have significant nonzero overlap with the rotational part of many wave functions having the same $J$ but different $n$ values. (The complete torsion-rotation wave functions are, of course, still orthogonal due to freerotor basis function orthogonality: $\left\langle n \mid n^{\prime}\right\rangle=\delta_{n, n^{\prime}}$.) Table II gives for the three states in Table I and Fig. 5 the number of rotational overlap matrix elements larger than 0.1 for torsion-rotation states corresponding to seven different $n$ values. The excess overlaps resulting from $K$ mixing (i.e., total overlaps minus seven) is 76 for the $K$-scrambled (elliptical trajectory) state E1, 39 for the state E2 corresponding classically to stable rotation around the north pole maximum, and only 10 for the state E3 corresponding to stable rotation around the south pole maximum.

Figure 6 shows the total number of overlaps greater than 0.1 between all rotational states in the $n=13$ free-rotor manifold and all rotational states in the $n^{\prime}=16$ free-rotor manifold, for acetaldehyde rotational constants and three sets of modified values for $\rho$ and $D_{a b}$. If there were no change in rotational state composition with $n$, the number of overlaps would be equal to $2 J+1$, which exactly describes the nearly coincident pair of straight lines for $\rho=0$ and $\rho=D_{a b}=0$ at the bottom of the figure. In principle, the maximum possible number of overlaps greater than 0.1 could increase as $(2 \mathrm{~J}$ $+1)^{2}$ until $J$ becomes large enough that severe $K$ scrambling reduces many overlaps below 0.1 . Such behavior is not apparent in Fig. 6, but the upper curve, corresponding to experimental acetaldehyde values for $\rho$ and $D_{a b}$, deviates approximately quadratically from the minimum straight line for $10<J<20$, and then reaches a saturation value of $3.7(2 J$ +1 ) for higher $J$.

We interpret the curves in Fig. 6 as follows. (i) There are no $K$-scrambling excess overlaps in the free-rotor model of acetaldehyde if one artificially sets $\rho=D_{a b}=0$. There is certainly $K$ mixing caused by $B-C \neq 0$, but this traditional asymmetric-rotor mixing does not, in general, change appreciably with $n$ and therefore does not lead to more than the minimum number of $(2 J+1)$ nonzero $\left\langle n ; J K \mid n^{\prime} ; J K^{\prime}\right\rangle$ rotational overlap integrals. (ii) The same is true if $\rho=0$ and $D_{a b} \neq 0$, since again the $K$ mixing does not change with $n$. (iii) If $\rho \neq 0$ and $D_{a b}=0$, some $K$-scrambling effects are observed, leading to approximately $0.7(2 J+1)$ excess overlaps for $J>30$. (iv) If $\rho$ and $D_{a b}$ are given their experimental values for acetaldehyde, more $K$ scrambling is observed,

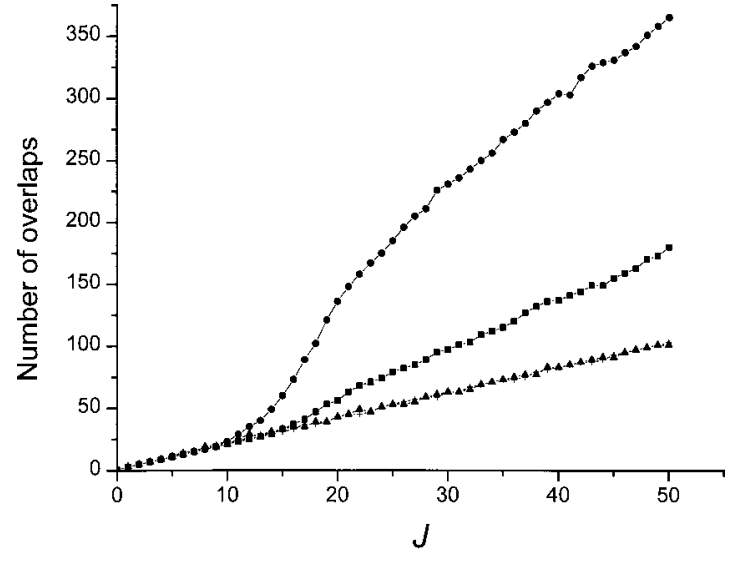

FIG. 6. The number of overlap integrals greater than 0.1 between rotational states of given $J$ in the $n=13$ free-rotor manifold and rotational states of the same $J$ in the $n^{\prime}=16$ manifold, calculated using $A, B, C$ rotational constants for acetaldehyde. The straight line at the bottom of the figure is a superposition of curves for $\rho=D_{a b}=0(+)$ and $\rho=0, D_{a b}=-0.123 \mathrm{~cm}^{-1}(\boldsymbol{\Delta})$. This line increases as $2 J+1$, and corresponds to only one overlap greater than 0.1 per rotational function in each of the two free-rotor manifolds, i.e., corresponds to essentially identical rotational functions in each of the two manifolds. The curve at the top ( ) calculated with $\rho=0.3291$ and $D_{a b}$ $=-0.123 \mathrm{~cm}^{-1}$ (the experimental acetaldehyde values), shows a delayed onset of $K$ scrambling, but for $J>25$ shows 3.7 times more overlap integrals greater than 0.1 than are found for $\rho=0$. The middle curve $(\boldsymbol{\square})$, with $\rho$ $=0.3291$ and $D_{a b}=0$ shows intermediate behavior. This figure in toto demonstrates that $K$-scrambling-induced excess overlap integrals require a nonzero $\rho$ value (i.e., require nonzero torsion-rotation Coriolis coupling), and are significantly enhanced by a nonzero $D_{a b}$ value (i.e., by a noncoaxial rotor).

leading to approximately $2.7(2 J+1)$ excess overlap integrals for $J>20$. These excess overlap integrals greater than 0.1 will lead to numerous perturbations and less numerous perturbation-allowed transitions in spectral assignments and analyses. Their potential significance for intramolecular vibrational relaxation is discussed in Sec. IV.

Finally, Fig. 7 shows the total number of overlap integrals greater than 0.1 as a function of $J$ for several values of $n$ and $n^{\prime}$. As expected, $K$ scrambling begins at lower $J$ for lower $n$ values, since smaller asymmetric-rotor forces are then required to overcome the tendency of the internal rotor to keep $K$ a good quantum number. As also expected, larger values of $n^{\prime}-n$ give rise to a larger number of excess overlap integrals, since the change in $K$ scrambling is larger for larger $n^{\prime}-n$.

\section{ACETALDEHYDE STATES NEAR THE FREE- ROTOR LIMIT}

In most real molecules with threefold barriers, it is not practical to study torsional levels high enough in energy to fully reach the free-rotor limit. We have, however, found many torsion-rotation states somewhat above the barrier in acetaldehyde that are well described by a single $e^{i n \alpha}$ freerotor function. These states are in the near-free-rotor limit, where $n$ is not a rigorously good quantum number because of small $\Delta n= \pm 3$ and \pm 6 mixings induced by the $V_{3}$ and $V_{6}$ barrier terms in Eq. (2). In this section we conclude that the qualitative free-rotor results of Sec. II can be applied essentially without change to the near-free-rotor case. 

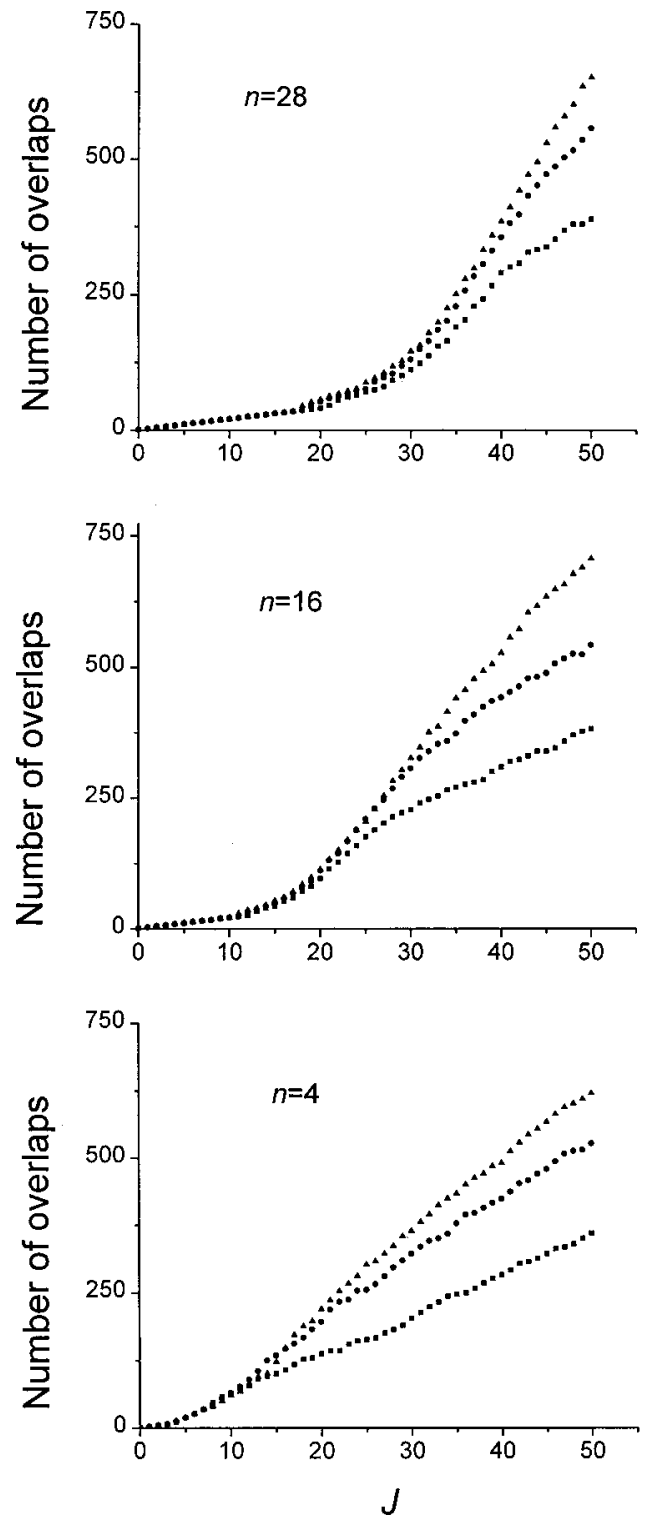

FIG. 7. The number of overlaps greater than 0.1 between rotational functions in various pairs of free-rotor torsional states $\left(n, n^{\prime}\right)$, calculated using acetaldehyde torsion-rotation constants. The $n$ value is given in the figure and $n^{\prime}=n+3(\mathbf{\square}), n^{\prime}=n+6(\bullet)$, and $n^{\prime}=n+9(\mathbf{\Delta})$. The increase in the number of overlaps over the minimum value of $2 J+1$, i.e., the onset of $K$-scrambling effects, is pushed to higher and higher $J$ values as $n$ increases, indicating that stronger and stronger rotational forces are required to counter the $K$-preserving tendencies of the free internal rotor. Also, more overlaps occur when the two free-rotor manifolds differ more in internal-rotation angular momentum, since larger $\Delta n$ values lead to larger differences in $K$ mixing.

Figure 8 shows the number of states that have reached the near-free-rotor limit in acetaldehyde as a function of $J$ for several $n$ values (based on a criterion of more than $80 \%$ contribution to the whole wave function from a single $n$ ). The straight line proportional to $2 J+1$ represents the maximum possible value for this number. Figure 8 indicates that (i) the free-rotor limit is reached more easily for high $n$ and low $J$, where effects of the barrier and of overall rotation on the internal rotation motion are relatively smaller, and (ii) a significant fraction of states above the barrier (i.e., with $n$ $\geqslant 5$ ) have reached the near-free-rotor limit.

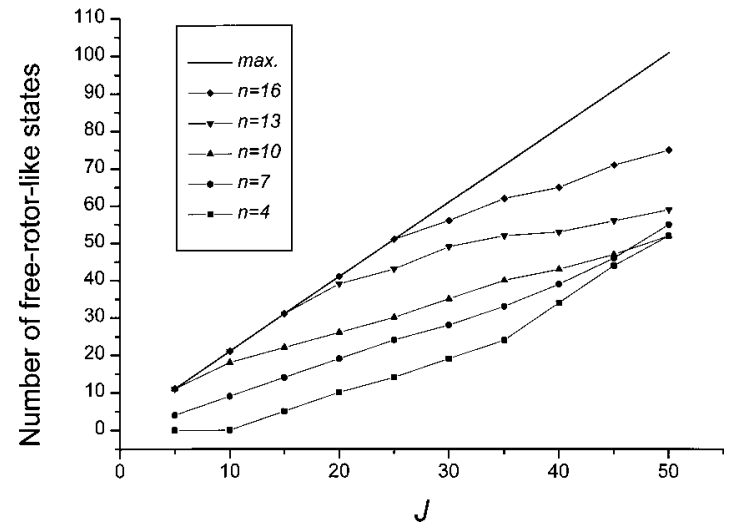

FIG. 8. The number of near-free-rotor states in acetaldehyde (i.e., states containing more than $80 \%$ of one particular $n$ value) shown as a function of $J$ for several $n$ values (all corresponding to states of $E$ symmetry species). The straight line proportional to $2 J+1$ at the top of the figure indicates the upper bound for this number.

Some of these nearly pure $n$ states show the same highly mixed rotational composition as found in the free-rotor case. This is easily understood because the cause of $K$ scrambling is not removed by effects of the barrier. In fact, mixing would, in general, be expected to increase when a $K$-scrambled state belonging to one $n$ gets mixed by the barrier with a rotational state belonging to a different $n$. We have examined a number of acetaldehyde near-free-rotor $|J K\rangle$ states for $n=13$ and 16 , and $J$ values up to 50 . The calculations with barrier mixings included are considerably larger and more time consuming than for the free-rotor case because of the large Hamiltonian matrices, and for that reason we have not done as exhaustive a numerical search as for the free-rotor limit discussed in the previous section. Nevertheless, we have found a number of $K$-scrambled states. For example, the $J=25$ eigenstate at $E=1770.166 \mathrm{~cm}^{-1}$ has $91 \% n=16$ character and in the original RAM axis system there are 13 basis functions contributing more than $1 \%$ (from $K=9$ to $K=24$ ). We thus conclude that even after taking into account the effects of the barrier there are many states in acetaldehyde whose $K$-scrambling behavior is qualitatively similar to that of free-rotor states.

Note that setting $\rho=0$ eliminates the excess overlap integrals associated with $K$ scrambling even when $V_{3} \neq 0$, since $\rho=0$ removes all coupling between the torsional problem and the rotational problem in the Hamiltonian of Eq. (1), and therefore removes all dependence of $K$ scrambling on the torsional state.

\section{DISCUSSION}

Experimental evidence ${ }^{20,21}$ indicates that intramolecular vibrational energy redistribution (see Refs. 22 and 23 for reviews on IVR) is accelerated in molecules that contain an internally rotating methyl group (i.e., a methyl top with torsional excitation approaching or exceeding the internal rotation barrier height). However, no unified theory capable of quantitatively explaining this enhancement exists at the moment (possibly because a number of mechanisms act synergistically). 
Martens and Reinhardt ${ }^{24}$ proposed that chaotic coupling between the methyl rotor and the lowest-frequency ring modes in $p$-fluorotoluene promotes vibrational state mixing and consequent energy transfer. For other kinds of molecules other mechanisms have been proposed. Li, Ezra, and Philips ${ }^{16}$ found that one source of coupling leading to enhanced IVR is vibrationally induced rotational axis switching (VIRAS). Their formalism is based on the physical picture that the rotation of body-fixed axes caused by vibrational excitation must be particularly important when large vibrational displacements are caused or stabilized by centrifugal distortion forces. The consequence, when their formalism is applied to $\mathrm{CH}_{2} \mathrm{~F}-\mathrm{CH}_{2} \mathrm{OH},{ }^{16}$ is that rotational wave functions for different torsional states have very different $K$ compositions. Purely anharmonic interaction terms in the potential energy surface are then able to couple one rotational level of a given zeroth-order vibration-torsion state with many rotational levels in some other vibration-torsion state. In this way VIRAS, which is similar to axis-switching effects in electronic transitions, ${ }^{25}$ could be a contributing factor to the IVR enhancement observed when internal rotors are present in a molecule.

The $K$-scrambled states for various free-rotor manifolds, as discussed in this paper, are also strongly nonorthogonal to many rotational states of other torsional states. However, no significant rotation of principal axes with torsional excitation occurs in our Hamiltonian (i.e., there is no significant VIRAS), since the rotational constants for a molecule with a methyl top do not change appreciably as the top rotates. It is rather the combined effects of the term $-2 F \rho n J_{a}$ and the terms $D_{a b}\left(J_{a} J_{b}+J_{b} J_{a}\right)$ and $\frac{1}{2}(B-C)\left(J_{b}^{2}-J_{c}^{2}\right)$ in our Hamiltonian that are responsible for the $K$ scrambling here. (As noted earlier, the latter two terms are responsible for any actual $K$ mixing, but the former term must also be present for the generation of excess overlap integrals, since otherwise the mixing would be the same for every torsional state.)

Consideration of Fermi's Golden Rule for the decay constant of a bright state $\Gamma=2 \pi\left\langle W^{2}\right\rangle \rho$ indicates that the effect of $K$-scrambled states on IVR is not obvious, since any increase in the density of coupled states $\rho$ could be compensated by a dilution of the mean squared interaction matrix element $\left\langle W^{2}\right\rangle$, leaving the product unchanged. Even though, as is well known, relaxational decay of a bright state does not replace quantum beats until the density of bath states is high enough for the statistical limit to be reached, a simple example of three levels embedded in the quasicontinuum can illustrate the possible enhancement of IVR by $K$ scrambling. Suppose that one bright state $\psi_{\mathrm{vt}} \psi_{r}$ is coupled to a distant bath state $\phi_{\mathrm{vt}} \phi_{r 1}$ and a nearly resonant bath state $\phi_{\mathrm{vt}} \phi_{r 2}$ by the same vibration-torsion term in the Hamiltonian $H_{\mathrm{vt}}$, i.e., consider the two matrix elements:

$$
\begin{aligned}
& \left\langle\psi_{\mathrm{vt}}\left|H_{\mathrm{vt}}\right| \phi_{\mathrm{vt}}\right\rangle\left\langle\psi_{r} \mid \phi_{r 1}\right\rangle, \\
& \left\langle\psi_{\mathrm{vt}}\left|H_{\mathrm{vt}}\right| \phi_{\mathrm{vt}}\right\rangle\left\langle\psi_{r} \mid \phi_{r 2}\right\rangle .
\end{aligned}
$$

When there is no $K$ scrambling, so that rotational wave functions in a given $J$ manifold are essentially independent of the associated torsion-vibration wave function, then only one of the rotational overlap integrals can be nonzero. If it is the overlap with the distant state in Eq. (4a), then a negligible contribution to the effective coupling out of the bright state is obtained. On the other hand, when $K$ scrambling is present, then both rotational overlap integrals can be nonzero, and a significant contribution from the near-resonant bath state in Eq. (4b) will be obtained. A generalization of this mechanism to a large number of states in the quasicontinuum could lead to a net increase in the product $\left\langle W^{2}\right\rangle \rho$, and thus also in the IVR rate. Such a mechanism predicts that IVR contributions arising from $K$ scrambling should depend nonmonotonically on the amount of rotational excitation of the bright state, since torsionally dependent $K$-scrambled states exist only for a particular range of $J / n$ values.

\section{ACKNOWLEDGMENTS}

This work was supported in part by the Spanish DGES (Project No. PB96-0881) and the Division of Chemical Sciences, Office of Basic Energy Sciences, Office of Energy Research, U.S. Department of Energy. The authors are indebted to Dr. G. T. Fraser for helpful criticism of the manuscript.

${ }^{1}$ C. C. Lin and J. D. Swalen, Rev. Mod. Phys. 31, 841 (1959).

${ }^{2}$ J. T. Hougen, I. Kleiner, and M. Godefroid, J. Mol. Spectrosc. 163, 559 (1994).

${ }^{3}$ H. M. Pickett, J. Chem. Phys. 107, 6732 (1997).

${ }^{4}$ V. Szalay and J. Ortigoso, J. Chem. Phys. 109, 3911 (1998).

${ }^{5}$ V. Szalay, J. Mol. Spectrosc. 128, 24 (1988).

${ }^{6}$ J. T. Hougen, J. Mol. Spectrosc. 181, 287 (1997).

${ }^{7}$ I. Kleiner, J. T. Hougen, J.-U. Grabow, S. P. Belov, M. Yu. Tretyakov, and J. Cosléou, J. Mol. Spectrosc. 179, 41 (1996) and references therein.

${ }^{8}$ B. G. Sumpter, C. C. Martens, and G. S. Ezra, J. Phys. Chem. 92, 7193 (1988).

${ }^{9}$ J. T. Vivian, S. A. Lehn, and J. H. Frederick, J. Chem. Phys. 107, 6646 (1997).

${ }^{10}$ J. Ortigoso, Phys. Rev. A 54, R2521 (1996).

${ }^{11}$ W. G. Harter and C. W. Patterson, J. Chem. Phys. 80, 4241 (1984).

${ }^{12}$ J. Ortigoso and J. T. Hougen, J. Chem. Phys. 101, 2710 (1994).

${ }^{13}$ F. T. Arecchi, E. Courtens, R. Gilmore, and H. Thomas, Phys. Rev. A 6, 2211 (1972)

${ }^{14}$ C. C. Martens, J. Chem. Phys. 90, 7064 (1989).

${ }^{15}$ J. Ortigoso, I. Kleiner, and J. T. Hougen, J. Chem. Phys. 110, 11688 (1999).

${ }^{16}$ H. Li, G. S. Ezra, and L. A. Philips, J. Chem. Phys. 97, 5956 (1992).

${ }^{17}$ G. M. Nathanson and G. M. McClelland, J. Chem. Phys. 81, 629 (1984).

${ }^{18}$ G. M. Nathanson and G. M. McClelland, J. Chem. Phys. 85, 4311 (1986).

${ }^{19}$ H. Leipholz, Stability Theory (Wiley, New York, 1987).

${ }^{20}$ V. A. Walters, S. D. Colson, D. L. Snavely, K. B. Wiberg, and B. M. Jamison, J. Phys. Chem. 89, 3857 (1985).

${ }^{21}$ C. S. Parmenter and B. M. Stone, J. Chem. Phys. 84, 4710 (1986).

${ }^{22}$ K. K. Lehmann, G. Scoles, and B. H. Pate, Annu. Rev. Phys. Chem. 45, 241 (1994).

${ }^{23}$ D. J. Nesbitt and R. W. Field, J. Phys. Chem. 100, 12735 (1996).

${ }^{24}$ C. C. Martens and W. P. Reinhardt, J. Chem. Phys. 93, 5621 (1990).

${ }^{25}$ J. T. Hougen and J. K. G. Watson, Can. J. Phys. 43, 298 (1965). 